\title{
Paradoxical vocal cord motion causing stridor after thyroidectomy
}

\author{
Joseph Harbison, Jonathan Dodd, Walter T McNicholas
}

\section{Abstract}

Two women developed stridor immediately after thyroidectomy as a result of paradoxical vocal cord motion. In both cases the cord function showed a normal pattern during vocalisation but paradoxical movement was seen at laryngoscopy during tidal breathing. The abnormality improved in both patients over time with speech therapy. Whilst the syndrome of paradoxical vocal cord motion is classically thought to have a largely psychological aetiology, subtle interference with

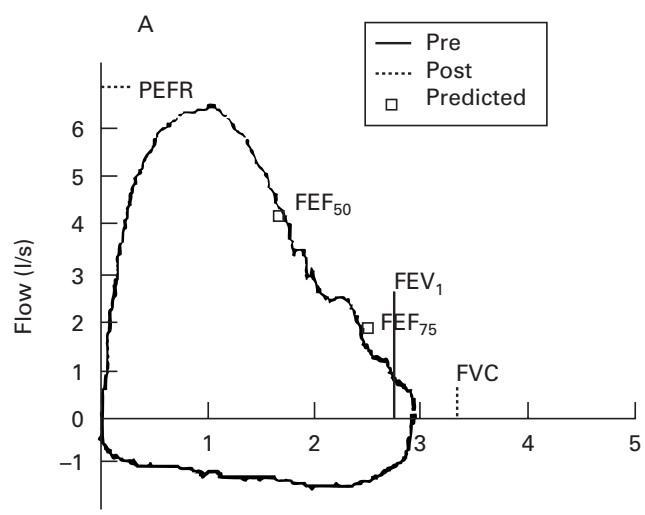

laryngeal innervation at surgery is more likely to have been the cause in these cases. (Thorax 2000;55:533-534)

Keywords: paradoxical vocal cord motion; thyroidectomy

Paradoxical vocal cord motion syndrome is an uncommon disorder characterised by inappropriate adduction of the vocal cords during inspiration instead of the normal abduction. ${ }^{1-3}$ We describe two cases who developed paradoxical vocal cord motion syndrome after thyroid surgery.
Department of

Respiratory Medicine, St Vincent's University Hospital, Dublin 4, Ireland

J Harbison

J Dodd

W T McNicholas

Correspondence to: Dr W T McNicholas email:wmcnicho@svherc.ucd.ie

Received 8 December 1998 Returned to authors 26 February 1999

Revised manuscript received

2 June 1999

Accepted for publication

15 June 1999
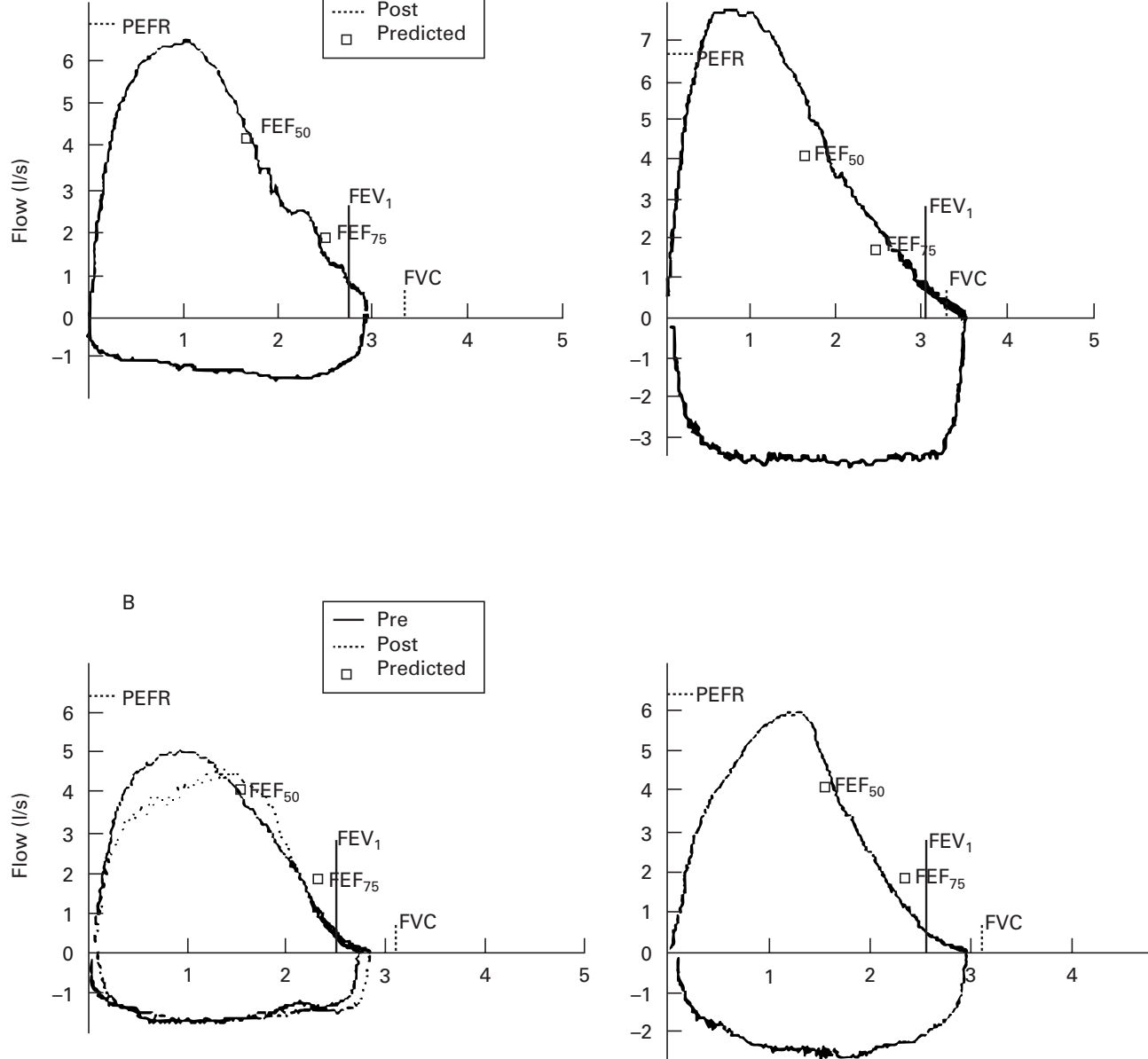

Figure 1 Flow-volume loops in (A) case 1 and (B) case 2 with postoperative findings on the left and three month follow up findings after treatment on the right. The loops demonstrate inspiratory flow limitation initially which improved on follow up, particularly in case 1. 


\section{Case reports}

CASE 1

A 47 year old woman with a large goitre and thyrotoxicosis had uneventful subtotal thyroidectomy performed under general anaesthesia. She had no significant medical or psychiatric history and did not smoke. In the recovery room she developed severe dyspnoea and stridor requiring re-intubation. Exploration of the wound was performed but no abnormalities were found. Extubation again led to stridor and she was re-intubated and transferred to the intensive care unit. Although stridor persisted on extubation 24 hours later, the patient was not in distress and was transferred to the ward. Examination revealed marked stridor during forced inspiratory effort without any obvious change in her voice quality. Pulmonary function tests revealed typical features of upper airway obstruction with limitation of maximal inspiratory flow rates (peak inspiratory flow rate (PIFR) $36 \%$ predicted) but normal expiratory flow (fig 1A, left). Fibreoptic laryngoscopy under intravenous midazolam sedation revealed a full range of vocal cord movements which, although normal during vocalisation, were paradoxical during tidal breathing. The patient was given intensive speech therapy and recovered over three months. Pulmonary function improved with PIFR rising to $79 \%$ (fig $1 \mathrm{~A}$, right) and repeat laryngoscopy revealed normal vocal cord motion.

CASE 2

A 37 year old woman with a history of right recurrent laryngeal nerve palsy following previous partial thyroidectomy required surgery for a large recurrent multinodular goitre. Before surgery she complained of occasional morning wheeze but was on no treatment. She was a non-smoker. Preoperative indirect laryngoscopy confirmed right vocal cord paralysis but normal left vocal cord movement. She underwent uneventful subtotal thyroidectomy but 24 hours later complained of dyspnoea and stridor was noted both when awake and asleep. Fibreoptic laryngoscopy under intravenous midazolam sedation showed paradoxical movements of the left vocal cord during tidal breathing which were normal during vocalisation. Pulmonary function testing revealed inspiratory flow limitation (PIFR 36\%) but normal expiratory flow rates (fig $1 \mathrm{~B}$, left). The patient was given speech therapy and both her symptoms and pulmonary function tests improved over the subsequent three months with PIFR rising to $55 \%$ (fig $1 \mathrm{~B}$, right), the residual inspiratory flow limitation probably reflecting previous damage to the right recurrent laryngeal nerve.

\section{Discussion}

These cases differ from the typical presentation of paradoxical vocal cord motion syndrome which usually occurs in middle aged women, often employed in a paramedical profession, many of whom have co-existing asthma, and the condition is suspected to have a largely psychological aetiology. ${ }^{1-3}$ In these two cases paradoxical cord motion may have been a consequence of temporary palsy of the recurrent laryngeal nerve due to surgical manipulation, and local trauma to the larynx during surgery and endotracheal intubation may also have contributed. Adduction of the vocal cords during inspiration is likely to have been secondary to the negative pressure developed during inspiration together with a venturi effect related to airflow. These cases also differ from the more typical presentation of recurrent laryngeal nerve palsy where hoarseness is the usual presenting symptom. ${ }^{4}$

Postoperative cases of paradoxical vocal cord motion have rarely been described in the past and have generally been attributed to psychological causes. ${ }^{5}{ }^{6}$ However, it would be difficult to attribute a psychological cause to either of these two cases. Notably, stridor was specifically observed during sleep which is atypical in functional stridor. The development of stridor immediately after thyroid surgery also suggests an organic cause.

Pulmonary function testing in cases of paradoxical cord motion shows marked inspiratory flow limitation but normal expiratory flow rates unless the patient has associated asthma. Laryngoscopy reveals paradoxical vocal cord movement with vocal cord adduction during inspiration and abduction during expiration. However, the paradoxical motion will only be detected if vocal cord movements are timed in conjunction with the phases of respiration.

The authors thank Mr John Hyland, consultant surgeon, who referred both cases to us.

1 Christopher KL, Wood RP, Eckert RC, et al. Vocal-cord dysfunction presenting as asthma. $N$ Engl $\mathcal{f}$ Med 1983;308:1566-70.

2 Newman KB, Mason UG, Schmaling KB. Clinical features of vocal cord dysfunction. Am $\mathcal{F}$ Respir Crit Care Med 1995; 152:1382-6.

3 Elshami M, Tino G. Coexistent asthma and functional upper airway obstruction. Case reports and a review of the literature. Chest 1996;110:1358-61.

4 Pracy R, Siegler J, Stell PM. A short textbook of ENT. 2nd ed. London: Hodder and Stoughton, 1974: 121-5.

5 Michelsen LG, Vanderspek AF. An unexpected functional cause of upper airway obstruction. Anaesthesia 1988;43: 1028-30.

6 Arndt GA, Voth BR. Paradoxical vocal cord motion in the recovery room: a masquerader of pulmonary dysfunction. Can F Anaesth 1996;43:1249-51. 